\title{
An extensive library of synthetic spectra covering the far red, RAVE and GAIA wavelength ranges
}

\author{
T. Zwitter ${ }^{1}$, F. Castelli ${ }^{2,3}$, and U. Munari ${ }^{4,5}$ \\ 1 University of Ljubljana, Department of Physics, Jadranska 19, 1000 Ljubljana, Slovenia \\ ${ }^{2}$ CNR - Istituto di Astrofisica Spaziale e Fisica Cosmica, Via del Fosso del Cavaliere, 00133 Roma, Italy \\ 3 INAF - Osservatorio Astronomico di Trieste, Via G.B. Tiepolo 11, 34131 Trieste, Italy \\ ${ }^{4}$ Osservatorio Astronomico di Padova, Sede di Asiago, 36012 Asiago (VI), Italy \\ 5 CISAS, Centro Interdipartimentale Studi ed Attività Spaziali dell’Università di Padova, Italy
}

Received 16 September 2003 / Accepted 24 December 2003

\begin{abstract}
A library of 183588 synthetic spectra based on Kurucz's ATLAS9 models is presented for the far red spectral interval (7653-8747 ̊). It is characterized by $3500 \mathrm{~K} \leq T_{\text {eff }} \leq 47500 \mathrm{~K}, 0.0 \leq \log g \leq 5.0,-3.0 \leq[\mathrm{M} / \mathrm{H}] \leq+0.5$, $0 \leq V_{\text {rot }} \leq 500 \mathrm{~km} \mathrm{~s}^{-1}, \xi=2 \mathrm{~km} \mathrm{~s}^{-1}$. The whole grid of spectra is calculated for resolving powers 8500, 11500 and 20000 . A section of the grid is also computed for $[\alpha / \mathrm{Fe}]=+0.4$ and for microturbulent velocities 0 and $4 \mathrm{~km} \mathrm{~s}^{-1}$. The library covers the wavelength ranges and resolutions of the two ambitious spectroscopic surveys by the ground experiment RAVE and the space mission GAIA. Cross-sections across the multi-dimensional data-cube are used to illustrate the behaviour of the strongest spectral lines. Interpretation of real data will have to include interpolation to grid substeps. We present a simple estimate of the accuracy of such a procedure.
\end{abstract}

Key words. astronomical data bases: miscellaneous - stars: fundamental parameters - surveys - stars: atmospheres

\section{Introduction}

Observations in the far red interval (7650-8750 $\mathrm{A})$ are becoming increasingly used for determination of basic stellar parameters. The advantages of this interval are its low sensitivity to reddening, high photon budget for late-type stars, good sensitivity of modern CCD detectors, and above all richness of its spectral features.

The far red interval includes several important spectral lines (Munari 1999): (a) the K I infrared resonant doublet (7664.907 $\AA, 7698.979 \AA)$; (b) the Na I non-resonant doublet $(8183.256 \AA, 8194.821 \AA)$; $(c)$ he O I triplet (8446.247, $8446.359,8446.758 \AA)$; $(d)$ the Fe I multiplets 60,401 and others with excitation potentials between 2.17 and $5.00 \mathrm{eV} ;(e)$ the head of the Paschen series of hydrogen, and, most importantly; $(f)$ the Ca II non-resonant infrared triplet (8498.018, $8542,089,8662.140 \AA$ ). The latter is present in all spectral types between $\mathrm{B} 8$ and $\mathrm{M}$. A further advantage is that the interval of 8400 to $8750 \AA$ is nearly free from telluric absorptions (Munari 1999).

The Ca II triplet is a powerful diagnostic tool: the strength of Ca II lines depends on metallicity, but it is essentially insensitive to age of stellar population (Garcia-Vargas et al. 1998; Schiavon et al. 2000; Vazdekis et al. 2003). These results have

Send offprint requests to: T. Zwitter,

e-mail: tomaz.zwitter@fmf.uni-lj.si been used to study behaviour of Ca II in composite stellar populations and to attempt to disentangle their age and metallicity. Composite systems include normal elliptical galaxies (Molla \& Garcia-Vargas 2000; Saglia et al. 2002; Cenarro et al. 2003), dwarf ellipticals (Michielsen et al. 2003) and active galaxies (van Groningen 1993; Diaz et al. 1989; Nelson \& Whittle 1999; Schinnerer et al. 2001; Marquez et al. 2003). Cenarro et al. (2002) studied the behaviour of Ca II line-strength indices in terms of effective temperature, surface gravity, and metallicity. Their analysis was based on a set of 706 real spectra. A much larger synthetic spectral library presented in this paper can be used to complement these results on $\mathrm{Ca}$ II and other lines in the red domain.

Two of the largest forthcoming spectral surveys are centered on the far red spectral interval. GAIA is the cornerstone 6 mission of ESA, approved for a launch around 2010. It is aimed at providing micro-arsec astrometry and $\sim 10$-band photometry for $\sim 10^{9}$ stars brighter than $V=20$. Brighter targets $(V<17.5)$ will also be observed by an on-board spectroscopic instrument operating in the $8480-8747 \AA$ wavelength range at a resolving power $R \equiv \lambda / \Delta \lambda=11500$ (Katz 2003). The other survey, Radial Velocity Experiment (RAVE), has just started in April (Steinmetz 2003). This is an international collaboration which uses the UK-Schmidt telescope at the Anglo Australian Observatory (AAO) equipped with a fiberoptic spectrograph to obtain spectra in a similar wavelength 
domain (8410-8750 $\AA$ ) and at a resolving power $R \sim 8500$. The goal is to observe 35 million stars brighter than $V=16$ at declinations suitable for AAO. The primary motivation for both surveys is to obtain stellar radial velocities to be used in studies of Galactic kinematics. But a cross-correlation with a library of stellar spectra with known values of physical parameters can yield much more than radial velocity: in fact the effective temperature, gravity, metallicity (with certain element abundances), rotational velocity, and the presence of different kinds of peculiarities can all be determined. Therefore any software development for the GAIA mission as well as analysis of the first RAVE data needs to use an extensive library of stellar spectra in the far red domain. The same holds for individual spectroscopic studies in this wavelength range which are becoming ever more numerous owing to the preparation of the GAIA mission (see e.g. Munari et al. 2001; Zwitter et al. 2003; Marrese et al. 2004).

Systematic observations of MKK standard stars in the far red spectral domain were presented by Munari \& Tomasella (1999) and by Marrese et al. (2003). It is difficult however to obtain high-signal-to-noise-ratio observations to map all relevant combinations of stellar parameters in a uniform manner. So a parallel effort was launched to calculate a grid of synthetic spectra using ATLAS9 models from Kurucz. The first two papers (Munari \& Castelli 2000; Castelli \& Munari 2001) explored the grid in the temperature-gravity-metallicity space for non-rotating stars, assuming a micro-turbulent velocity of $2 \mathrm{~km} \mathrm{~s}^{-1}$. Altogether 952 spectra were presented.

These early works were aimed to coarsely but rapidly explore an essentially unknown wavelength range to assist early planning and instrument design for GAIA. This phase is now over, and the community requirements are now moving toward data and analysis reduction pipelines. A much more extended and complete grid is now essential, and to provide one is the aim of this paper. The grid made available with this paper should meet the community requirements in testing algorithm coding for some time to come. The one to be used to analyze the actual GAIA data when they will be finally assembled around 2015-2018 will be computed only in the next decade taking full advantages of the continuous advacements in the input physics, coding and atomic constants that will be reached by that time. The present grid is also timely presented to assist with analysis of RAVE spectra that are already routinely obtained at AAO.

The present grid extends earlier calculations by adding more spectra, inclusion of stellar rotation and presentation of results at different spectral resolving powers. Furthermore a limited number of spectra corresponding to enhanced $\alpha$-element abundances and different values of micro-turbulent velocity are presented. The database consists of more than 183500 spectra and is freely available in electronic form at the CDS via anonymous ftp to cdsarc.u-strasbg.fr $(130.79 .128 .5)$ or via http://cdsweb.u-strasbg.fr/cgi-bin/qcat?J/A+A/417/1055 as well as via the ESA webserver (http://gaia.esa.int/ spectralib/).
Table 1. Properties of models at different metallicities. The first three columns give values of metallicity, $\alpha$-enhancement coefficient and microturbulent velocity $(\xi)$. The last two columns state if the "noovershooting" approximation to the convection treatment and if new opacity distribution functions were used.

\begin{tabular}{rcccc}
\hline \hline$[\mathrm{M} / \mathrm{H}]$ & {$[\alpha / \mathrm{Fe}]$} & $\xi\left[\mathrm{km} \mathrm{s}^{-1}\right]$ & NOVER & ODFNEW \\
\hline+0.5 & 0.0 & 2 & yes & no \\
+0.2 & 0.0 & 2 & no & no \\
0.0 & 0.0 & 0 & yes & no \\
0.0 & 0.0 & 2 & yes & no \\
0.0 & 0.0 & 4 & yes & no \\
-0.2 & 0.0 & 2 & no & no \\
-0.5 & 0.0 & 2 & yes & no \\
-0.5 & 0.4 & 2 & yes & yes \\
-1.0 & 0.0 & 2 & yes & no \\
-1.0 & 0.4 & 2 & yes & yes \\
-1.5 & 0.0 & 2 & yes & no \\
-1.5 & 0.4 & 2 & yes & yes \\
-2.0 & 0.0 & 2 & yes & no \\
-2.0 & 0.4 & 2 & yes & no \\
-2.5 & 0.0 & 2 & yes & no \\
-3.0 & 0.0 & 2 & no & no \\
-4.0 & 0.0 & 2 & no & no \\
\hline
\end{tabular}

\section{Computation of synthetic spectra}

We computed synthetic spectra for the 7650-8750 ̊ interval for almost all ATLAS9 grids of model atmospheres with different metallicities and micro-turbulent velocities available at the Kurucz web-site (http://kurucz.harvard.edu). We generally used the NOVER models which differ from the previous Kurucz (1993a) models for the convection in that they were recomputed by Castelli with the overshooting option for the convection switched off (Castelli et al. 1997). When NOVER grids were not available, we used the Kurucz (1993a) models. Because new ATLAS9 models based on updated Opacity Distribution Functions (ODFNEW models) have been recently computed (Castelli \& Kurucz 2003), we used them for a few metallicities with $T_{\text {eff }} \geq 5000 \mathrm{~K}$ and for all the spectra with $T_{\text {eff }} \leq 4750 \mathrm{~K}$. In fact, the main difference between the NOVER models and the ODFNEW models is the use of new Opacity Distribution Functions (ODFs) computed with TiO lines from Schwenke (1998) instead of from Kurucz, and including $\mathrm{H}_{2} \mathrm{O}$ lines, not considered at all in the previous ODFs. Also the ODFNEW models computed up to now are available at the Kurucz web-site (ODFNEW grids). Details of the adopted models are given in Table 1.

The synthetic spectra were computed with the SYNTHE code of Kurucz (1993b) at a resolving power of 500000 . The source of atomic data was Kurucz \& Bell (1995), the source of molecular data, except TiO, was Kurucz (1993c), while the source of TiO data was Kurucz (1999a), who supplied Schwenke's (1998) computations in SYNTHE format. Di-atomic molecules used in model computation are discussed in Kurucz (1992) and Kurucz (1993d). The source for 
Table 2. General ranges of parameters of the spectra. Not all combinations were calculated (for details see Figs. 1-3).

\begin{tabular}{lrlll}
\hline \hline Parameter & \multicolumn{2}{c}{ Range } & Step or Values \\
\hline Temperature & 3500 & $\ldots$ & $47500 \mathrm{~K}$ & step $250 \mathrm{~K}\left(T_{\text {eff }} \leq 10000 \mathrm{~K}\right), 500$ or $1000 \mathrm{~K}\left(T_{\text {eff }}>10000 \mathrm{~K}\right)$ \\
Metallicity & +0.5 & $\ldots$ & -3.0 & step $0.5 \&$ values of +0.2 and -0.2 \\
Gravity & 0.0 & $\ldots$ & 5.0 & step 0.5 \\
$\alpha$-enhancement & 0.0 & $\ldots$ & 0.4 & $(0.0,0.4)$ \\
Micro-turbulence & 0 & $\ldots$ & $4 \mathrm{~km} \mathrm{~s}^{-1}$ & $\left(0,2,4 \mathrm{~km} \mathrm{~s}^{-1}\right)$ \\
Rotation velocity & 0 & $\ldots$ & $100 \mathrm{~km} \mathrm{~s}^{-1}\left(T_{\text {eff }}<7000 \mathrm{~K}\right)$ & $\left(0,2,5,10,15,20,30,40,50,75,100 \mathrm{~km} \mathrm{~s}^{-1}\right)$ \\
& 0 & $\ldots$ & $500 \mathrm{~km} \mathrm{~s}^{-1}\left(T_{\text {eff }} \geq 7000 \mathrm{~K}\right)$ & $\left(0,10,20,30,40,50,75,100,150,200,250,300,400,500 \mathrm{~km} \mathrm{~s}^{-1}\right)$ \\
Resolving power & 8500 & $\ldots$ & 20000 & $(8500,11500,20000)$ \\
Wavelength & 7653 & $\ldots$ & $8747 \AA$ & 2 pixels per resolution element $(2.5$ pixels at $R=20000)$ \\
\hline
\end{tabular}

$\mathrm{H}_{2} \mathrm{O}$ data is Partridge \& Schwenke (1997), as distributed by Kurucz (1999b). The solar abundances are from Anders \& Grevesse (1989), except for the spectra based on the ODFNEW models. In this case the solar abundances are from Grevesse \& Sauval (1998). Each spectrum computed for a given model atmosphere was then broadened for several values of rotational velocity $V_{\text {rot }}$ and for three resolving powers: $R=8500,11500$, and 20000 by assuming a Gaussian instrumental profile. These resolutions were chosen because they correspond to the baselined values for the RAVE survey, the GAIA mission and a typical Cassegrain-fed Echelle spectrograph, respectively. The resulting final synthetic spectra were resampled to 2 pixels per resolution element $(R=8500,11500)$ or 2.5 pixels per resolution element $(R=20000)$.

\section{Grid of synthetic spectra}

Ranges and steps for all seven basic parameters of the grid of synthetic spectra are given in Table 2 . We adopt a common convention of quoting metallicity and enhancement of $\alpha$-elements in logarithmic units with respect to the solar values. The gravity is in logarithmic cgs units. Details of all calculated parameter combinations are given in Figs. 1-3. Spectra are placed in gravity-temperature planes, with metallicity coded by a symbol type. Figure 1 covers the most numerous spectra, i.e. the ones with no $\alpha$-enhancement and with microturbulent velocity of $2 \mathrm{~km} \mathrm{~s}^{-1}$. The computed spectra cover the whole gravitytemperature plane except for hot low-gravity models which are not radiatively stable. Low-temperature spectra $\left(T_{\text {eff }}<5000 \mathrm{~K}\right)$ were computed for a sparser set of metallicities due to large requirements of computing time. These spectra will be added online when completed. Figure 2 corresponds to $\alpha$-enhanced cases and Fig. 3 to those with a different value of microturbulent velocity. Note that each of the symbols actually corresponds to $11\left(T_{\text {eff }}<7000 \mathrm{~K}\right)$ or $14\left(T_{\text {eff }} \geq 7000 \mathrm{~K}\right)$ spectra with different values of rotational velocity (see Table 1 ) and to three different resolving powers.

All spectra are available as ascii files grouped into different directories according to their resolving power and temperature. The filenames are in a standard format identified in Table 3. So f765875v010r20000m05t05250g45k2noverasc corresponds to a flux calibrated spectrum between 7650 and $8750 \AA$, with $V_{\text {rot }}=10 \mathrm{~km} \mathrm{~s}^{-1}, \lambda / \Delta \lambda=20000,[\mathrm{M} / \mathrm{H}]=$ $-0.5, T_{\text {eff }}=5250 \mathrm{~K}, \log g=4.5, \xi=2 \mathrm{~km} \mathrm{~s}^{-1}$, and no $\alpha$-enhancement.

The calculated grid is by far too large to present all of its properties here, so we explore only sample cross-sections across the grid. Figure 4 is a greyscale presentation of the spectra which were normalized to enhance line visibility. Each panel shows variation along one parameter axis, starting from a spectrum of a non-rotating K0 V type star. Note that all spectra were calculated in a wider wavelength domain, but only the 8400-8750 A range is plotted for clarity.

The temperature panel of Fig. 4 clearly shows the importance of sharp Ca II lines for any radial velocity study. The panel is a textbook example of the expected behaviour of the Paschen lines and metallic lines. The metallicity panel illustrates that the $\mathrm{Ca}$ II lines remain strong even at the lowest metallicities and the gravity panel shows their presence in all luminosity classes. The rotational velocity and resolving power panels show how the lines get smeared at high rotational velocities or if observing at low resolving powers.

The steps in the calculated grid are relatively small, but the coverage is not continuous. As an example, the step in temperature is $250 \mathrm{~K}$ (for $T_{\text {eff }} \leq 10000 \mathrm{~K}$ ). This is larger than the baselined accuracy of temperature determination for both GAIA and RAVE surveys. So the grid will have to be interpolated to smaller steps. Figure 5 illustrates the errors introduced by a simple linear interpolation. At a certain grid point $i$ with the parameter values $p_{i}$ we compare the true synthetic spectrum $S\left(p_{i}\right)$ with the spectrum $S^{\prime}$ obtained from a linear combination of spectra at neighbouring grid points: $S^{\prime}=f_{i-1} S\left(p_{i-1}\right)+f_{i+1} S\left(p_{i+1}\right)$. The weights $f_{i-1}$ and $f_{i+1}$ are optimized so that $\int\left[S\left(p_{i}\right)-S^{\prime}\right]^{2} \mathrm{~d} \lambda$ is minimal. The difference between the interpolated values of parameters $p^{\prime}$ and the true ones $p_{i}$ can then be expressed in units of a grid step:

$\Delta \equiv \frac{p^{\prime}-p_{i}}{p_{i+1}-p_{i-1}}=\frac{f_{i+1}\left(p_{i+1}-p_{i}\right)+f_{i-1}\left(p_{i-1}-p_{i}\right)}{\left(f_{i+1}+f_{i-1}\right)\left(p_{i+1}-p_{i-1}\right)}$.

Figure 5 shows that linear interpolation is accurate to $\lesssim 10 \%$ of the grid step. Note that this is the worst case scenario, corresponding to a reconstruction of the spectrum at the middle of 


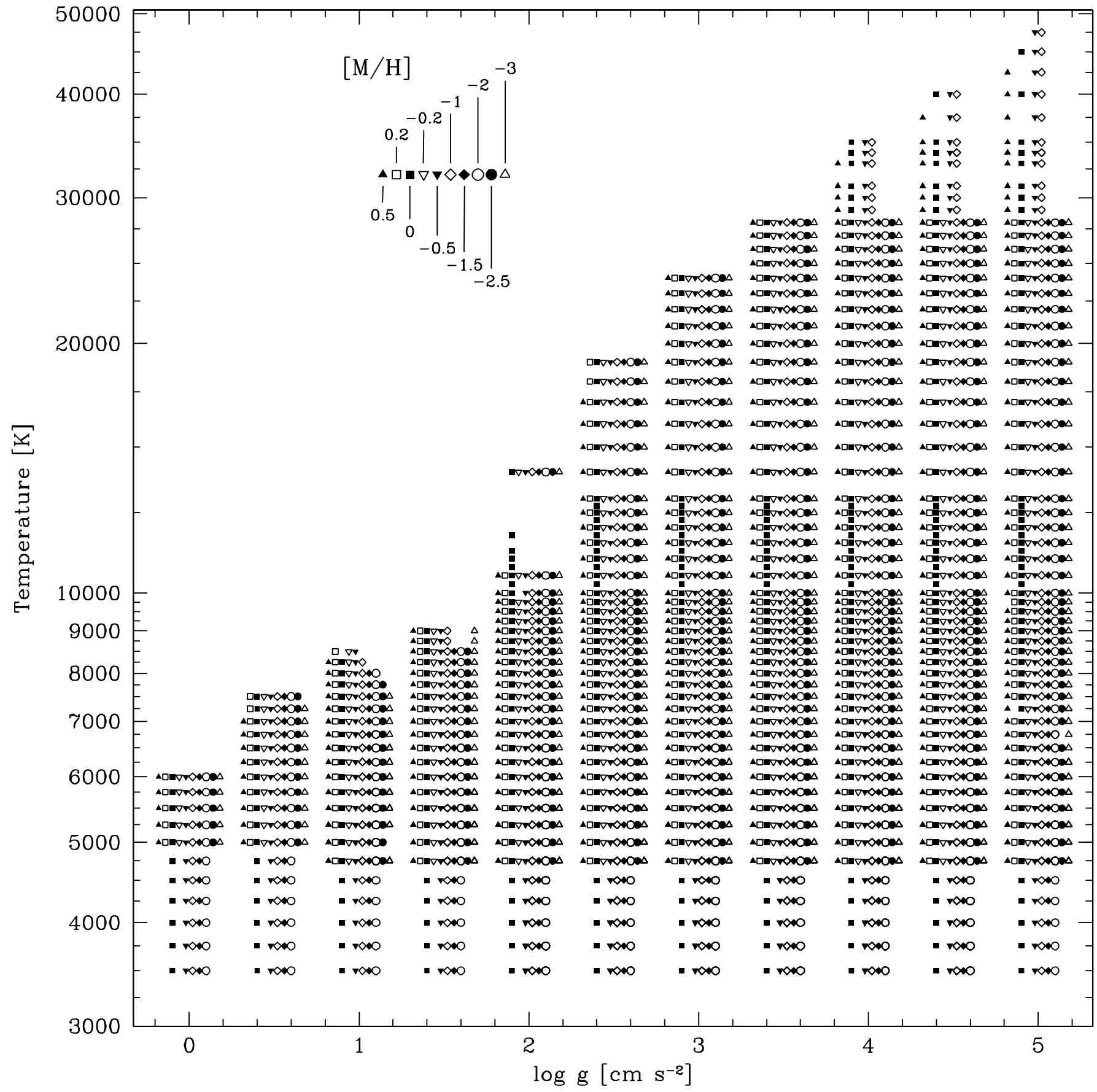

Fig. 1. Graph of calculated spectra without alpha enhancement and with a micro-turbulent velocity of $2 \mathrm{~km} \mathrm{~s}^{-1}$. Metallicity is coded with different symbols which are plotted with small horizontal offsets for clarity. Each spectrum is calculated for $11\left(T_{\text {eff }}<7000 \mathrm{~K}\right)$ or 14 ( $T_{\text {eff }} \geq$ $7000 \mathrm{~K}$ ) different rotational velocities and at three different resolving powers (see text).

the grid interval. Linear interpolation would be more accurate for spectra lying closer to one of the grid points. The results could be improved further by employing non-linear interpolation schemes. One may conclude that linear interpolation itself does not introduce errors exceeding $25 \mathrm{~K}$ in temperature (for $T_{\text {eff }}<10000 \mathrm{~K}$ ), $0.05 \mathrm{dex}$ in $[\mathrm{M} / \mathrm{H}]$ or $\log g$ and $1 \mathrm{~km} \mathrm{~s}^{-1}$ in $V_{\text {rot }}$. Note that other errors are more important: degeneracy of parameter values fitting spectra with a limited signal to noise ratio complicates their determination (Bailer-Jones 2003, see also Fig. 1 in Zwitter 2002). Also, spectra of real stars do not correspond exactly to the synthetic spectra due to their peculiarities (e.g. emission lines, varied abundances of individual elements, non-LTE effects, and non-static atmospheric structure).

\section{Discussion and conclusions}

The grid of synthetic spectra in the 7653-8747 $\AA$ interval presented here is the most extensive so far. Altogether the grid contains 183588 ascii files. They span three spectral 


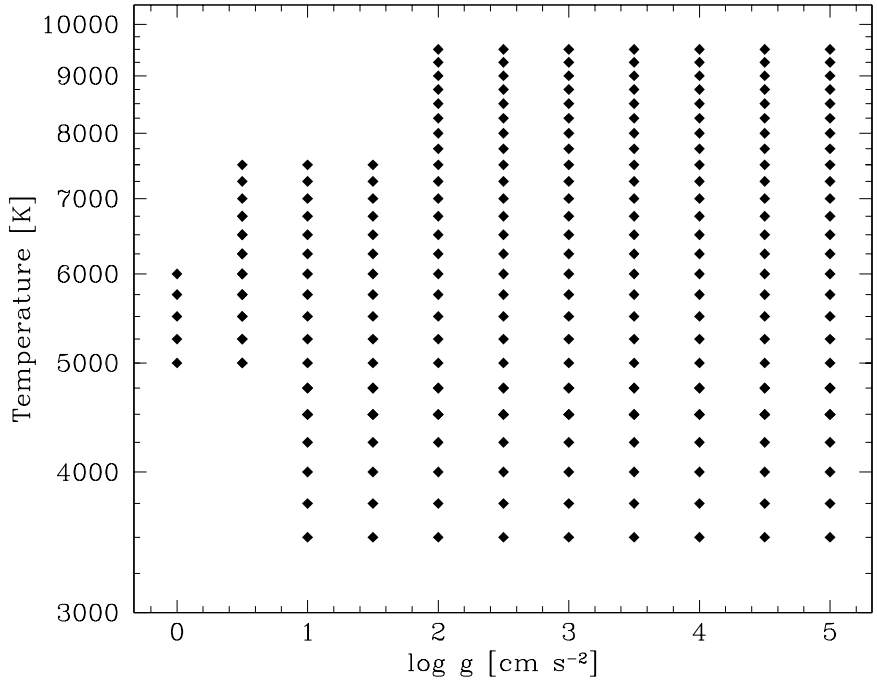

Fig. 2. As Fig. 1, but for models with alpha-enhancement $([\alpha / \mathrm{Fe}]=$ +0.4 ). All models were calculated for the metallicities $[\mathrm{M} / \mathrm{H}]=-0.5$, $-1.0,-1.5$, and -2.0 .

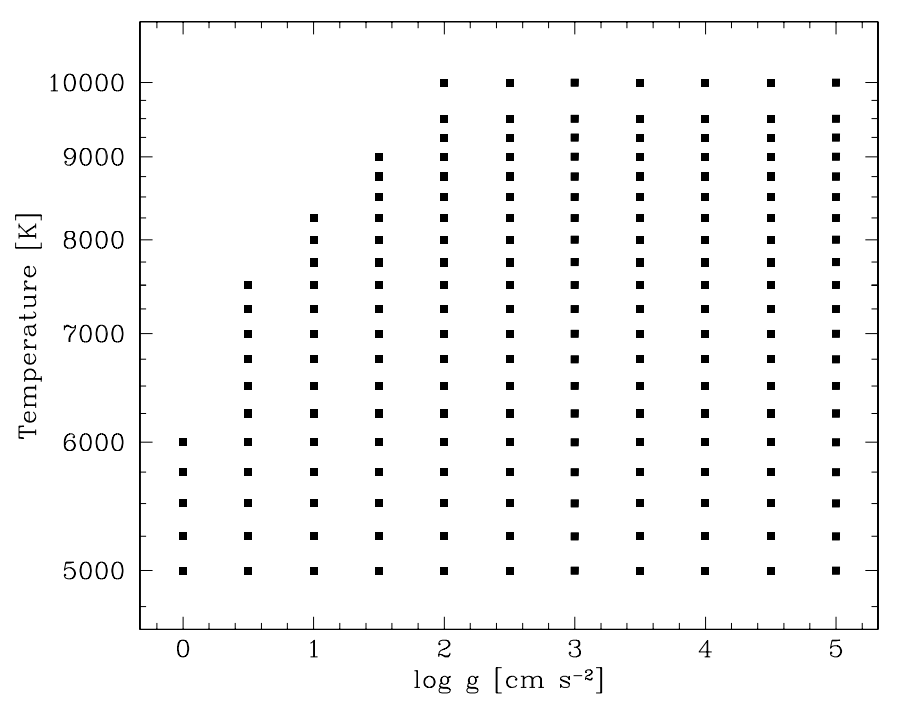

Fig. 3. As Fig. 1, but for models with micro-turbulent velocities of 0 and $4 \mathrm{~km} \mathrm{~s}^{-1}$. All models have solar abundances.

resolutions corresponding to a typical Echelle spectrograph and the spectrographs of the RAVE and GAIA surveys. This should facilitate comparison of the results obtained with different instruments. Spectra with other resolving powers $(R<20000)$ can be easily computed from the grid.

The synthetic spectra we have computed can be used as templates for the determination of radial velocity, the primary goal of the RAVE and GAIA surveys. They also permit the user to derive the primary parameters of stellar atmospheres: temperature, metallicity, gravity and rotational velocity. The grid includes subsets of spectra computed for +0.4 enhanced abundances of the $\alpha$ elements and for different values of the microturbulent velocity. It was shown that the grid can be easily interpolated to approximate spectra with parameter values between the steps in the grid. The errors of parameters computed with a
Table 3. Filenames of individual spectra. Meaning of corresponding characters is given.

\begin{tabular}{rl}
\hline \hline character & meaning \\
\hline 1 & f: fluxed spectrum $\left(\mathrm{erg} \mathrm{s}^{-1} \mathrm{~cm}^{-2} \AA^{-1}\right.$ steradian $\left.^{-1}\right)$ \\
& n: normalized to the continuum flux \\
$2-4$ & starting wavelength $(\mathrm{nm})$ \\
$5-7$ & ending wavelength $(\mathrm{nm})$ \\
$9-11$ & rotational velocity $V_{\text {rot }}\left(\mathrm{km} \mathrm{s}^{-1}\right)$ \\
$13-17$ & resolving power $R$ \\
18 & p: $[\mathrm{M} / \mathrm{H}] \geq 0.0$ \\
& m: $[\mathrm{M} / \mathrm{H}]<0.0$ \\
$19-20$ & $10 \times \mathrm{ABS}([\mathrm{M} / \mathrm{H}])$ \\
21 & $\mathrm{t}:$ no $\alpha$-enhancement \\
& a: $[\alpha /$ Fe $]=0.4$ \\
$22-26$ & effective temperature $T_{\text {eff }}(\mathrm{K})$ \\
$28-29$ & $10 \times \log g$ \\
31 & micro-turbulent velocity $\xi\left(\mathrm{km} \mathrm{s}^{-1}\right)$ \\
\hline
\end{tabular}

simple linear interpolation do not exceed $10 \%$ of the grid step. The computed spectra depend on the adopted values of individual element abundances. In particular the solar spectrum computed with Anders \& Grevesse's (1989) solar abundances for all the elements (Fig. 6) features too strong O I and Fe I lines. This is consistent with a recently suggested lower oxygen solar abundance $\log (N(\mathrm{O}) / N(\mathrm{H}))=-3.31$ dex (Prieto et al. 2001) and with a lower Fe I abundance $\log (N(\mathrm{Fe}) / N(\mathrm{H}))=-4.5 \mathrm{dex}$ (Bellot Rubio \& Borrero 2002) as obtained from two and three dimensional hydrodynamical model atmospheres. This complements classical methods of diagnostic line ratios for the far red spectral interval discussed by Munari (2002).

Properties of Kurucz ATLAS model atmospheres in the context of the red spectral interval and the GAIA mission have already been reviewed by Nesvacil et al. (2003). Plez (2003) estimated the potential of the MARCS models. Hauschildt et al. (2003) presented the computations of non-stationary and nonspherically symmetric atmospheres with significant amounts of dust. Finally Thévenin et al. (2003) reviewed the determination of chemical abundances from GAIA spectra. The common conclusion is that the observational potentials of the GAIA mission ask for improvements in the computation of synthetic stellar spectra. The RAVE collaboration is starting to obtain a very large number of high quality spectra in the far red spectral interval. They guarantee that this wavelength range is rapidly rising in importance. We are currently extending the grid presented here to a larger wavelength interval. A continuous coverage from $250 \mathrm{~nm}$ to $1050 \mathrm{~nm}$ should facilitate a comparison of spectra obtained in other wavelength domains to huge observational sets coming from the RAVE and GAIA surveys. 

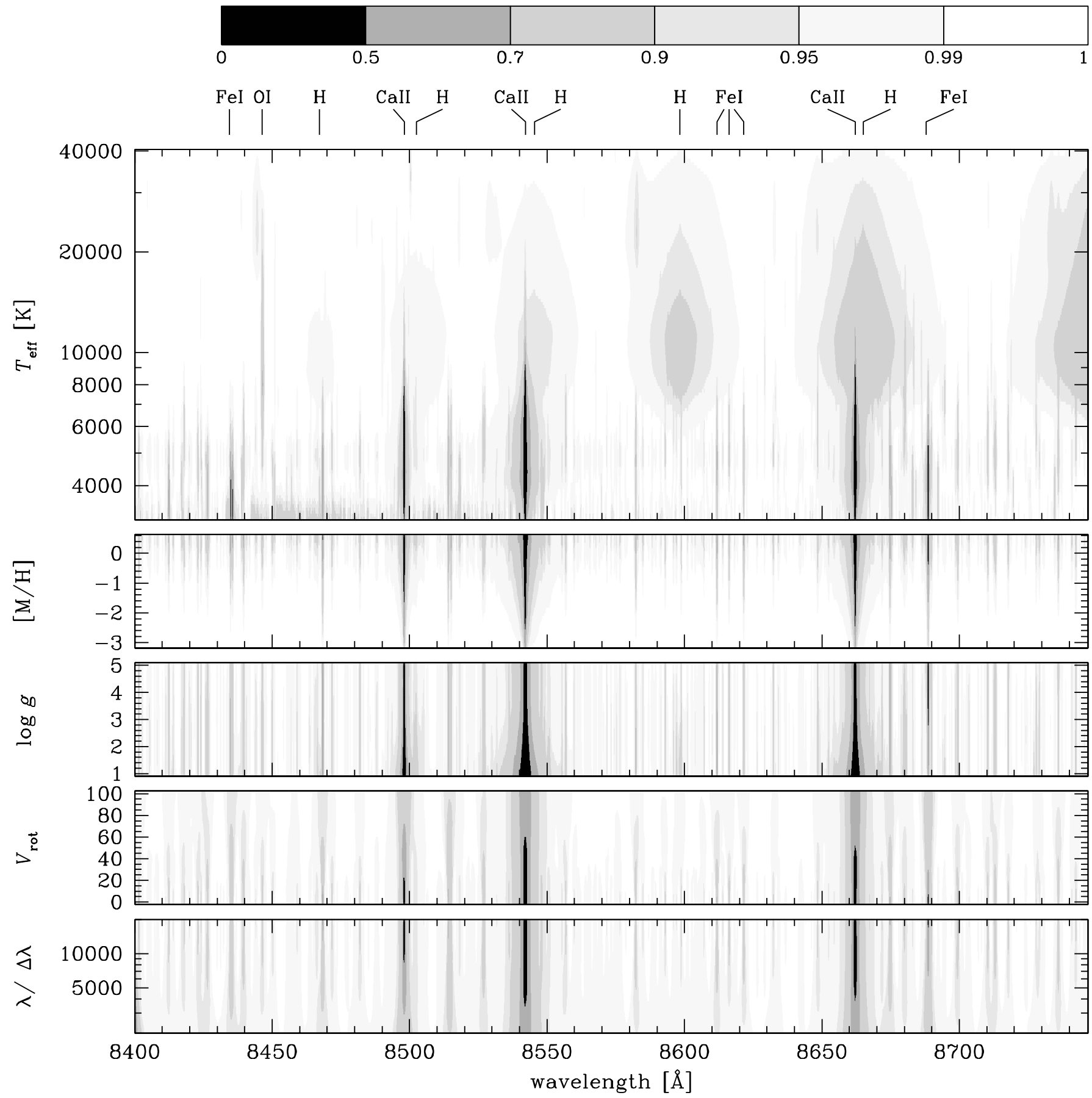

Fig. 4. Cross-sections through the multidimensional [Temperature, Metallicity, Gravity $\left(\mathrm{cm} \mathrm{s}^{-2}\right)$, Rotation, Resolving power] data-cube of computed spectra. Parameter values correspond to a non-rotating K0 V type star $\left(T_{\text {eff }}=5250 \mathrm{~K},[\mathrm{M} / \mathrm{H}]=0.0, \log g=4.5, V_{\text {rot }}=0 \mathrm{~km} \mathrm{~s}{ }^{-1}\right.$, $\left.\xi=2 \mathrm{~km} \mathrm{~s}^{-1}, \lambda / \Delta \lambda=20000\right)$, except for the parameter which is allowed to vary on a particular plot. All spectra were normalized by a single cubic spline fit to their upper envelope. The number of greyscale hues (identified at the top of the figure) is intentionally kept small, so as to allow reading the depth of individual spectral lines from the graph. Ions giving rise to the most prominent spectral lines are identified at the top. Synthetic spectra span a wider wavelength range and include variation of additional parameters ( $\alpha$-enhancement, different micro-turbulent velocities) not shown here for clarity.

Acknowledgements. We thank the anonymous referee for very relevant comments on the first version of the paper. T.Z. acknowledges financial support from the Slovenian Ministry for Education, Science and Sports, CNRS and the Royal Society as well as warm hospitality of GAIA groups at the Observatoire de Meudon and the
Mullard Space Sciences Laboratory where part of this work has been completed. U.M. acknowledges financial support from the Italian Space Agency contract ASI-I-R-117-01 and the Italian Ministry of Education COFIN 2001 grant. We thank R. Sordo for assisting with parts of the grid computation. 

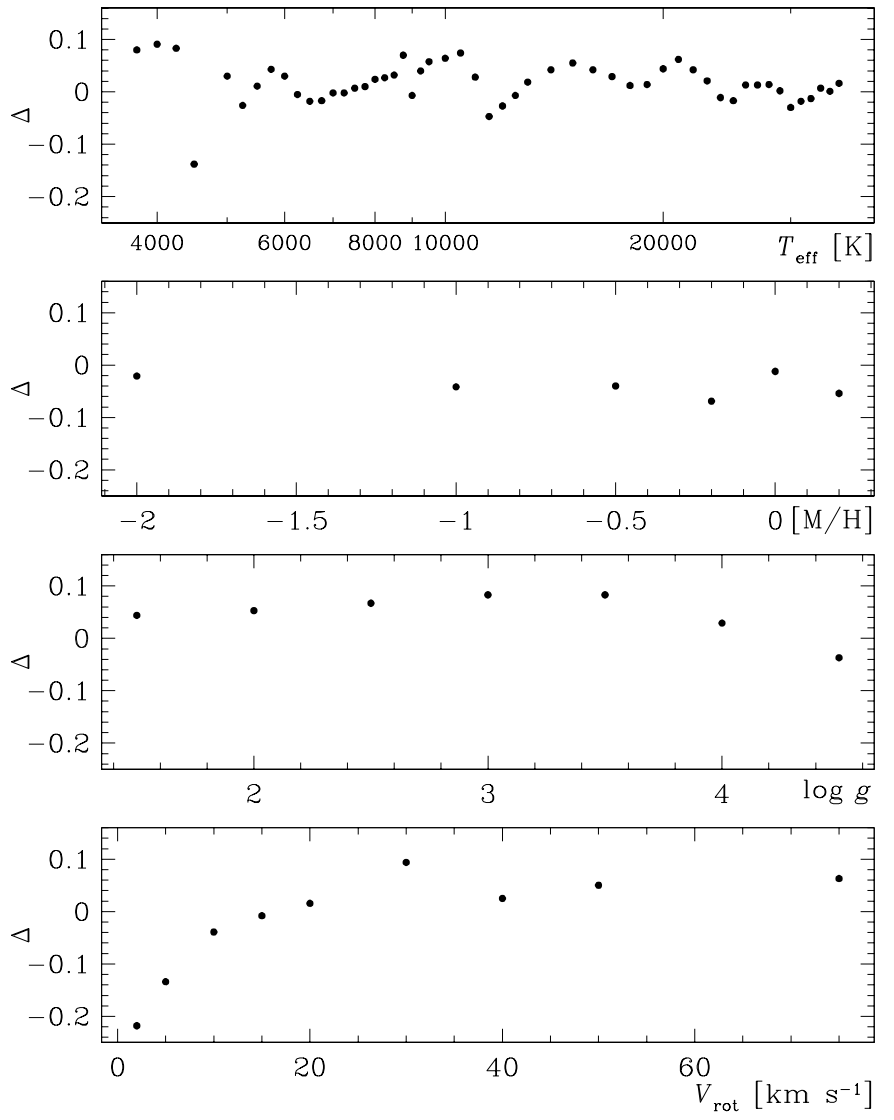

Fig. 5. Errors introduced by linear interpolation of the grid. Each graph explores variation of one parameter, with the values of other parameters being fixed at: $T_{\text {eff }}=5250 \mathrm{~K},[\mathrm{M} / \mathrm{H}]=0.0, \log g=4.5$, $V_{\text {rot }}=0 \mathrm{~km} \mathrm{~s}^{-1}, R=20000$. The ordinate is the difference between the value obtained from linear grid interpolation of the given parameter and the true value, expressed in units of the grid step. Note that linear interpolation yields values accurate to $\lesssim 10 \%$ of the grid step.

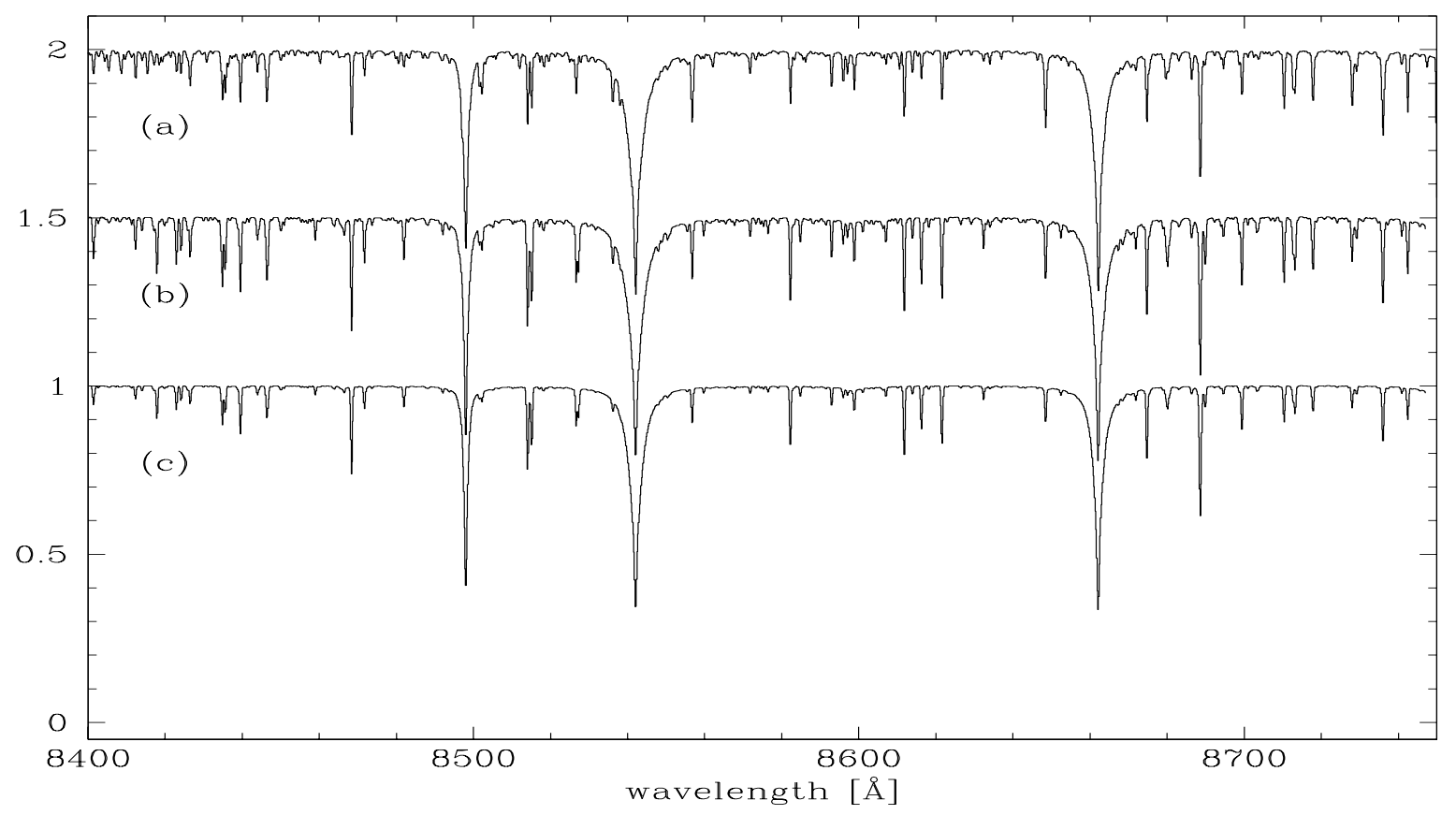

Fig. 6. Comparison of computed and observed spectra: a) observed solar flux atlas resampled to $R=20000$ (Kurucz et al. 1984); b) computed spectrum $\left(T_{\text {eff }}=5750 \mathrm{~K},[\mathrm{M} / \mathrm{H}]=0.0, \log g=4.5, V_{\text {rot }}=2 \mathrm{~km} \mathrm{~s}^{-1}, \xi=2 \mathrm{~km} \mathrm{~s}^{-1}, R=20000\right)$; c) same as b) but for $[\mathrm{M} / \mathrm{H}]=-0.5$. All spectra are plotted in normalized flux. The topmost two spectra have been vertically offset for clarity.

\section{References}

Anders, E., \& Grevesse, N. 1989, GeCoA, 53, 197

Bailer-Jones, C. A. L. 2003, in GAIA Spectroscopy, Science and Technology, ed. U. Munari, ASP Conf. Ser., 298, 199
Bellot Rubio, L. R., \& Borrero, J. M. 2002, A\&A, 391, 331

Castelli, F., Gratton, R. G., \& Kurucz, R. L. 1997, A\&A, 318, 841

Castelli, F., \& Munari, U. 2001, A\&A, 366, 1003

Castelli, F., \& Kurucz, R. L. 2003, in Modelling of Stellar Atmospheres, IAU Symp., 210, ed. N. E. Piskunov, et al., in press 
Cenarro, A. J., Gorgas, J., Cardiel, N., Vazdekis, A., \& Peletier, R. F. 2002, MNRAS, 329, 863

Cenarro, A. J., Gorgas, J., Vazdekis, A., Cardiel, N., \& Peletier, R. F. 2003, MNRAS, 339, L12

Diaz, A. I., Terlevich, E., \& Terlevich, R. 1989, in Active Galactic Nuclei, ed. D. E. Osterbrock, \& J. S. Miller, 560

Garcia-Vargas, M. L., Mollá, M., \& Bressan, A. 1998, A\&AS, 130, 513

Grevesse, N., \& Sauval, A. J. 1998, Space Sci. Rev., 85, 161

van Groningen, E. 1993, A\&A, 272, 25

Hauschildt, P. H., Allard, F., Baron, E., Aufdenberg, J., \& Schweitzer, A. 2003, in GAIA Spectroscopy, Science and Technology, ed. U. Munari, ASP Conf. Ser., 298, 179

Katz, D. 2003, in GAIA Spectroscopy, Science and Technology, ed. U. Munari, ASP Conf. Ser., 298, 65

Kurucz, R. L., Furenlid, I., Brault, J., \& Testerman, L. 1984, National Solar Observatory, Sunspot, New Mexico, Solar Flux Atlas from 296 to $1300 \mathrm{~nm}$

Kurucz, R. L. 1992, RvMexAA, 23, 45

Kurucz, R. L. 1993a, ATLAS9 Stellar Atmospheres Programs and $2 \mathrm{~km} \mathrm{~s}^{-1}$ grid, CD-ROM No. 13

Kurucz, R. L. 1993b, SYNTHE Spectrum Synthesis Programs and Line Data, CD-ROM No. 18

Kurucz, R. L. 1993c, Diatomic molecular data for opacity calculations, CD-ROM No. 15

Kurucz, R. L. 1993d, in Molecules in the Stellar Environment, IAU Coll., 146, ed. U. G. Jorgensen, 282

Kurucz, R. L., \& Bell, B. 1995, Atomic Line List, CD-ROM, No. 23

Kurucz, R. L. 1999a, TiO Line List, CD-ROM No. 24

Kurucz, R. L. 1999b, H2O Linelist from Partridge and Schwenke (1997), no IDs, CD-ROM No. 26

Marrese, P. M., Boschi, F., \& Munari, U. 2003, A\&A, 406, 995
Marrese, P. M., Munari, U., Siviero, A., et al. 2004, A\&A, 413, 635

Marquez, I., Masegosa, J., Durret, F., et al. 2003, A\&A, 409, 459

Michielsen, D., De Rijcke, S., \& Dejonghe, H. 2003, ApJ, 597, L21

Mollá, M., \& Garcia-Vargas, M. L. 2000, A\&A, 359, 18

Munari, U. 1999, Balt. A. 8, 73

Munari, U., \& Tomasella, L. 1999, 137, 521

Munari, U., \& Castelli, F. 2000, A\&AS, 141, 141

Munari, U., Tomov, T., Zwitter, T., et al. 2001, A\&A, 378, 477

Munari, U. 2002, in GAIA: A European Space Project, ed. O. Bienayme, \& C. Turon, EAS Publ. Ser., 2, 39

Nelson, C., \& Whittle, M. 1999, Adv. Space Res., 23, 891

Nesvacil, N., Stütz, C., \& Weiss, W. W. 2003, in GAIA Spectroscopy, Science and Technology, ed. U. Munari, ASP Conf. Ser., 298, 173

Partridge, H., \& Schwenke, D. W. 1997, J. Chem. Phys., 106, 4619

Plez, B. 2003, in GAIA Spectroscopy, Science and Technology, ed. U. Munari, ASP Conf. Ser., 298, 189

Prieto, C. A., Lambert, D. L., \& Asplund, M. 2001, ApJ, 556, L63

Saglia, R. P., Maraston, C., Thomas, D., Bender, R., \& Colless, M. 2002, ApJ, 579, L13

Schiavon, R. P., Barbuy, B., \& Bruzual, G. 2000, ApJ, 532, 453

Schinnerer, E., Eckart, A., \& Tacconi, L. J. 2001, ApJ, 549, 254

Schwenke, D. W. 1998, Chemistry and Physics of Molecules and Grains in Space, Faraday Discussions No. 109, The Faraday Division of the Royal Soc. of Chem., London, 321

Steinmetz, M. 2003, in GAIA Spectroscopy, Science and Technology, ed. U. Munari, ASP Conf. Ser., 298, 381

Thévenin, F., Bijaoui, A., \& Katz, D. 2003, in GAIA Spectroscopy, Science and Technology, ed. U. Munari, ASP Conf. Ser., 298, 291

Vazdekis, A., Cenarro, A. J., Gorgas, J., Cardiel, N., \& Peletier, R. F. 2003, MNRAS, 340, 1317

Zwitter, T. 2002, A\&A, 386, 748

Zwitter, T., Munari, U., Marrese, P. M., et al. 2003, A\&A, 404, 333 